\title{
Protective Effect of Valsartan on Beleomycine-Induced Fibrosis
}

\author{
Hoda Mojiri Forushani, ${ }^{1}$ Ali Asghar Hemmati, ${ }^{1,}$ Ali Khodadadi, ${ }^{2}$ Mohammad-Ali Assarehzadegan, ${ }^{3}$ \\ and Mohammad Rashno ${ }^{2}$ \\ ${ }^{1}$ Pharmacology Department,School of Pharmacy and Physiology Research Center, Ahvaz Jundishapur University of Medical Sciences, Ahvaz, Iran \\ ${ }^{2}$ Immunology Department, School of Medicine, Ahvaz Jundishapur University of Medical Sciences (AJUMS), Ahvaz, Iran \\ ${ }^{3}$ Immunology Department, School of Medicine, Iran University of Medical Sciences, Tehran, Iran \\ "Corresponding author: Ali Asghar Hemmati, Pharmacology Department, School of Pharmacy and Physiology Research Center, Ahvaz Jundishapur University of Medical \\ Sciences, Ahvaz, Iran. Tel: +98-6153532395, E-mail: hemmati-aa@yahoo.com
}

Received 2015 November 07; Revised 2016 May 13; Accepted 2016 May 29.

\begin{abstract}
Background: Pulmonary fibrosis (PF) is among the world's most prevalent and common respiratory system diseases. Several previous studies have revealed the contribution of angiotensin in the pathogenesis of PF; subsequently, angiotensin-converting enzyme inhibitors (ACEIs) and angiotensin receptor antagonists (ARDs) have been proposed for the treatment of pulmonary fibrosis using therapeutic approaches.

Objectives: This study aimed to investigate the anti-fibrotic effect of valsartan as an angiotensin receptor blocker.

Methods: Rats were given a single intratracheal administration of bleomycin (7.5 IU/kg); valsartan (20, 40, $80 \mathrm{mg} / \mathrm{kg} / \mathrm{day}) \mathrm{was}$ administrated to the rats orally, starting seven days before the induction of lung fibrosis and continuing until the end of the study. The control group received a vehicle.

Results: The rats that received valsartan exhibited decreased hydroxyprolin content and pulmonary index values. Pathological examination showed that valsartan could prevent inflammation and fibrotic scarring induced by bleomycin.

Conclusions: Valsartan displayed an anti-fibrotic and protective effect against bleomycin-induced lung fibrosis in an animal model.
\end{abstract}

Keywords: Valsartan, Pulmonary Fibrosis, Angiotensin, Bleomycin

\section{Background}

Pulmonary fibrosis (PF) is a complex, chronic condition that is generally considered to be the result of a variety of acute and chronic lung diseases. PF is characterized by increasing collagen content, inflammation, excessive matrix deposition, and destruction of the lung architecture, ultimately leading to respiratory failure (1).

Although some of the etiological aspects of PF remain unknown, cigarette smoking, viral infections, and surfactant protein polymorphisms have been proposed as risk factors for the progression of PF (2). To date, no standard treatments have been introduced in clinical settings, although glucocorticoids and cytotoxic drugs have been utilized (3). Death usually occurs as the result of respiratory complications; the survival rate is roughly 50 percent within five years $(4,5)$. Angiotensin-converting enzyme (ACE), via proteolytic actions on angiotensin I, produce angiotensin II. Angiotensin II (Ang II), as a bioactive peptide of the renin-angiotensin system (RAS), plays an important role in the initiation and maintenance of lung fibrosis (6). A growing body of evidence indicates that Ang II regulates the fibrotic response to tissue injury via the induction of the proliferation of human lung fibroblast, the production of lung procollagen (7-9), and the induction of alveolar ep- ithelial cell apoptosis by the binding of Ang II to the AT1 receptor $(8,10)$. Studies have also shown that angiotensinconverting enzyme inhibitors (ACEIs) (11) and Ang II type 1 receptor blockers (ARBs) (12) may be used for the management of PF in animal models and humans. Related studies in the literature have primarily concentrated on discovering the associated mechanisms through which these medications improve PF pathological indices.

In the current assessment, we chose to examine an angiotensin receptor blocker known as valsartan, which has been applied for several years in different clinical conditions, including controlling hypertension and heart failure (13), utilizing valsartan's presumptive actions in blocking the binding of Ang II to the Ang II type 1 receptor (AT1).

\section{Objectives}

The present research specified the anti-fibrotic and probable protective effects of valsartan as an angiotensin receptor antagonist for investigation; future cellular studies and other experiments will be required to determine the exact nature of valsartan's beneficial involvement in this process. 


\section{Methods}

\subsection{Animals}

Healthy male Sprague-Dawley (SD) rats weighing 200$220 \mathrm{~g}$ were purchased from the animal house and research center of Jundishapur University of Medical Sciences, Ahvaz, Iran. Animals were kept at $23 \pm 2{ }^{\circ} \mathrm{C}$ with an alternating cycle of $12 \mathrm{~h}$ light and dark. The rats had free access to food and tap water ad libitum. The animal experimental protocol used was according to the Jundishapur University Animal Care and Use Committee.

\subsection{Drug Treatment Groups}

On the basis of the primary evaluations and previous studies, the optimal doses of valsartan were selected $(20,40$, and $80 \mathrm{mg} / \mathrm{kg})$. The animals were randomly divided into five groups of eight rats each, as follows. The control group received a single intratracheal (IT) dose of bleomycin (BLM) solution (7.5 IU/kg), while the vehicle group was given a single PO dose of saline $(0.2 \mathrm{ml} / \mathrm{kg})$. Each treatment group received valsartan (Dr. Abidi Pharmaceutical Company, Iran) in doses of 20,40 , or $80 \mathrm{mg} / \mathrm{kg}$, seven days before administration of a single IT dose of BLM (7.5 $\mathrm{IU} / \mathrm{kg}$ ); treatments were continued for up to three weeks. The day for BLM (IT) injection was designated as the seventh day. Valsartan and saline were given once daily in the treatment and vehicle groups (respectively) for four weeks.

\subsection{Induction of Pulmonary Fibrosis by Bleomycin}

According to Schraufnagel et al.'s method (14), on the seventh day of treatment, the rats were anaesthetized with ketamine (50 $\mathrm{mg} / \mathrm{kg}$ ) intraperitoneally (IP), placed on a slanted board, and hung from their upper incisors. Keeping the nose of each animal closed and its tongue out, BLM solution $(7.5 \mathrm{IU} / \mathrm{kg})$ was administrated into the trachea via the mouth by a modified needle. After recovery from anesthesia, the rats were carefully returned to their cages.

\subsection{Isolation of Lung Tissue}

At the end of the treatment course, all rats were weighed. The animals were sacrificed, and their lungs were dissected out; the whole lung weight was then recorded. One part of each lung was fixed with 10 percent buffered formalin for histopathological evaluation; another part of each lung was separated for measurement of hydroxyproline. Lung specimens were stored at $-70^{\circ} \mathrm{C}$ until hedroxyproline assay could be conducted.

\subsection{Determination of Lung Index}

Each rat was weighed before sacrifice. The lungs were removed and weighed using an electronic balance; the lung index was expressed as the ratio of lung wet weight (mg) to body weight ( $\mathrm{g})$.

\subsection{Histological Evaluation}

Sections of fixed embedded tissues were cut to $4 \mu \mathrm{m}$ using a rotary microtome, and then the lung tissue sections were stained with hematoxylin and eosin (H\&E). Finally, all tissue sections were studied with light microscopy.

\subsection{Hydroxyproline Measurement in Lung Tissue}

Hydroxyproline is considered to be a good index of collagen deposition. Lung samples were homogenized with homogenizer and then hydrolyzed in $6 \mathrm{~N} \mathrm{HCl}$ for 18 hours at $110^{\circ} \mathrm{C} ; 2.5 \mathrm{M} \mathrm{NaOH}$ was then added to neutralize the samples. Chloramine $\mathrm{T}(1 \mathrm{~mL})$, perchloric acid (1 $\mathrm{mL})$, and dimethylaminobenzaldehyde $(1 \mathrm{~mL})$ were added to the samples. The absorbance of the samples were read to be $550 \mathrm{~nm}$ in a spectrophotometer. The results were expressed as milligrams of hydroxyproline (HP) per gram of lung tissue (15).

\subsection{Statistical Analysis}

The data are presented as the mean $+\mid$ - scanning electron microscope (SEM) values. Statistically significant differences between groups were determined by ANOVA followed by Tukey's test. Significance was accepted when $\mathrm{P}<$ 0.05 .

\section{Results}

\subsection{Effect of Valsartan on the Lung Index}

An increase in the lung index is one of the indices representing lung inflammation. As shown in Figure 1, lung index values decreased significantly in comparison to the BLM group; the lung index values significantly increased in the BLM-induced PF model in comparison to the control group.

\subsection{Effect of Valsartan on Hydroxyproline Content}

As shown in Figure 2, the hydroxyproline content in the lung tissues was significantly increased in the BLMexposed groups; valsartan in the three different doses (20, $40,80 \mathrm{mg} / \mathrm{kg}$ ) markedly decreased the hydroxyproline content in comparison to the BLM group.

\subsection{Effect of Valsartan on Alveolar Inflammation and Lung Fi- brogenesis}

The histopathological findings of the lung tissues showed that for the control group, alveolar spaces, alveolar septa, and lung structure were normal, and no lesions were evident (Figure 3A). The administration of bleomycin precipitated significant histological changes, including detectable areas of inflammatory infiltration, increases in 
Figure 1. Relationship Between Lung Weight and Body Weight

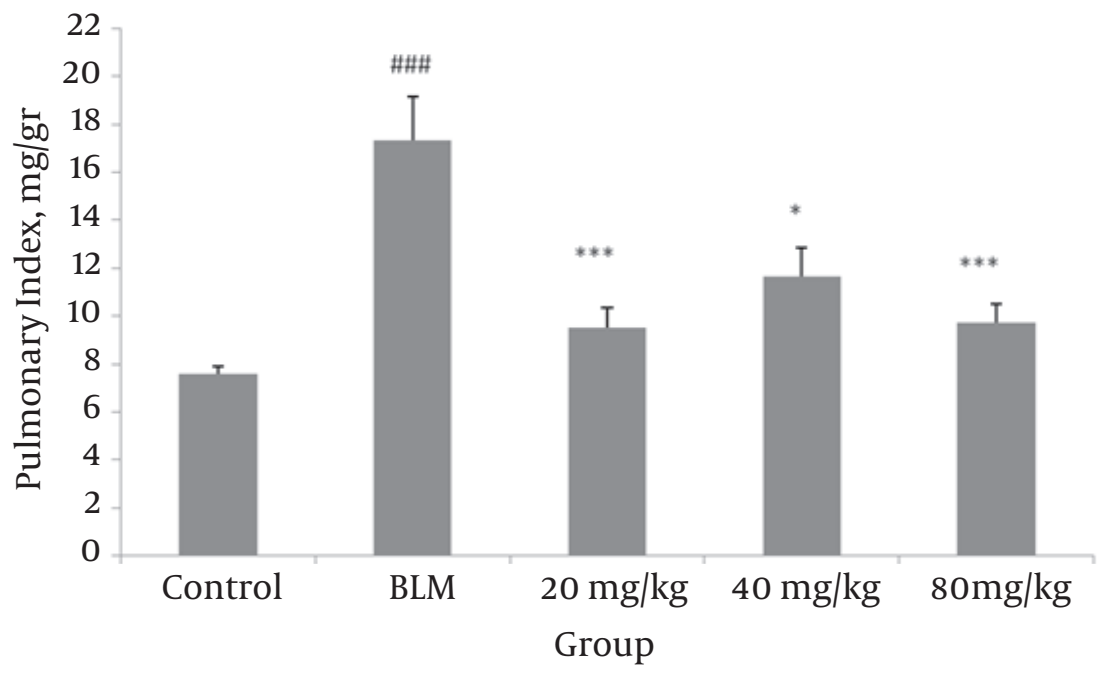

A significant increase was observed in the lung/body weight relationship after bleomycin (BLM) administration (\#\#\# P<0.001 vs. the control group). In different doses of valsartan, the pulmonary index values were significantly decreased vs. the BLM group $\left({ }^{*} \mathrm{P}<0.05 ;{ }^{* * *} \mathrm{P}<0.001\right)$.

Figure 2. Effect of Valsartan on the Hydroxyproline Content in Rat Lungs with Pulmonary Fibrosis

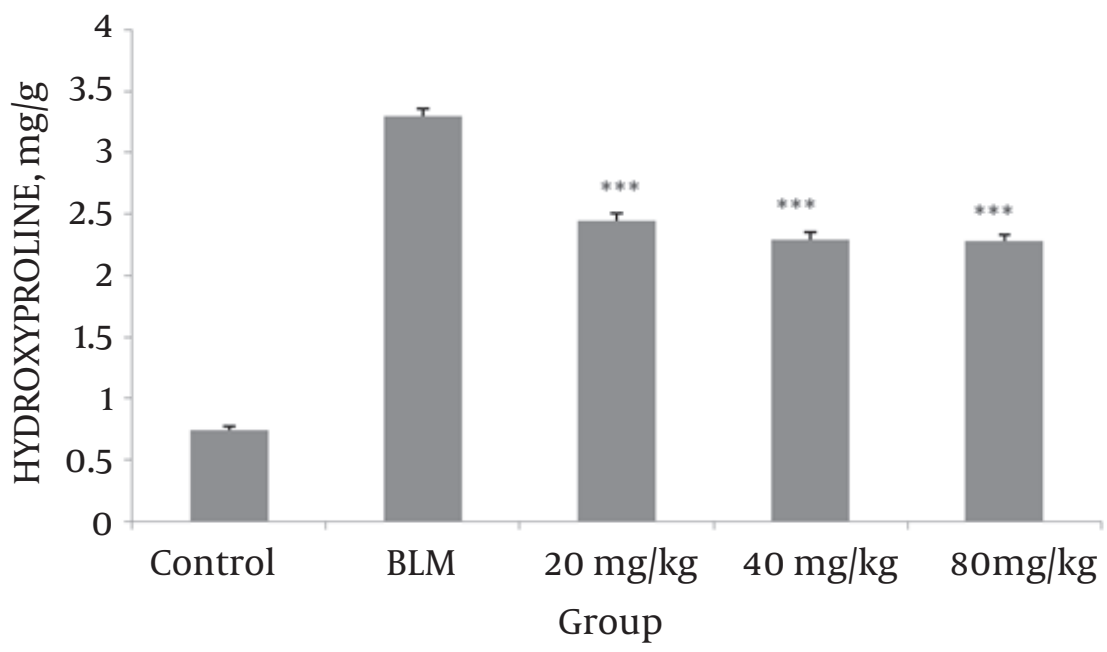

Hydroxyprolin content in the three different doses of valsartan significantly decreased in comparison to the BLM group $\left({ }^{* * *} \mathrm{P}<0.001\right)$.

the thickness of the alveolar walls, and collagen deposition and fibroblastic manifestations (Figure 3B). Adding valsartan in different doses showed significantly reductive changes in lung morphology: less inflammation, less cell infiltration, and less collagen deposition and septal widening were observed (Figure 3C, D, E). Pathological changes were observed, however, among the three doses of valsar$\tan (20,40,80 \mathrm{mg} / \mathrm{kg})$; the severity of the changes differed from slight to moderate.

\section{Discussion}

Pulmonary fibrosis is a progressive fatal lung disease (1). No satisfactory therapeutic agent has been found, and the current protocols have not indicated their expected efficacy nor their possibly severe adverse effects. A growing body of studies have thus been undertaken to find novel and effective ways to improve the management of the consequences of this fatal condition $(5,16)$. Most studies to date have used the bleomycin-induced pulmonary lesion 

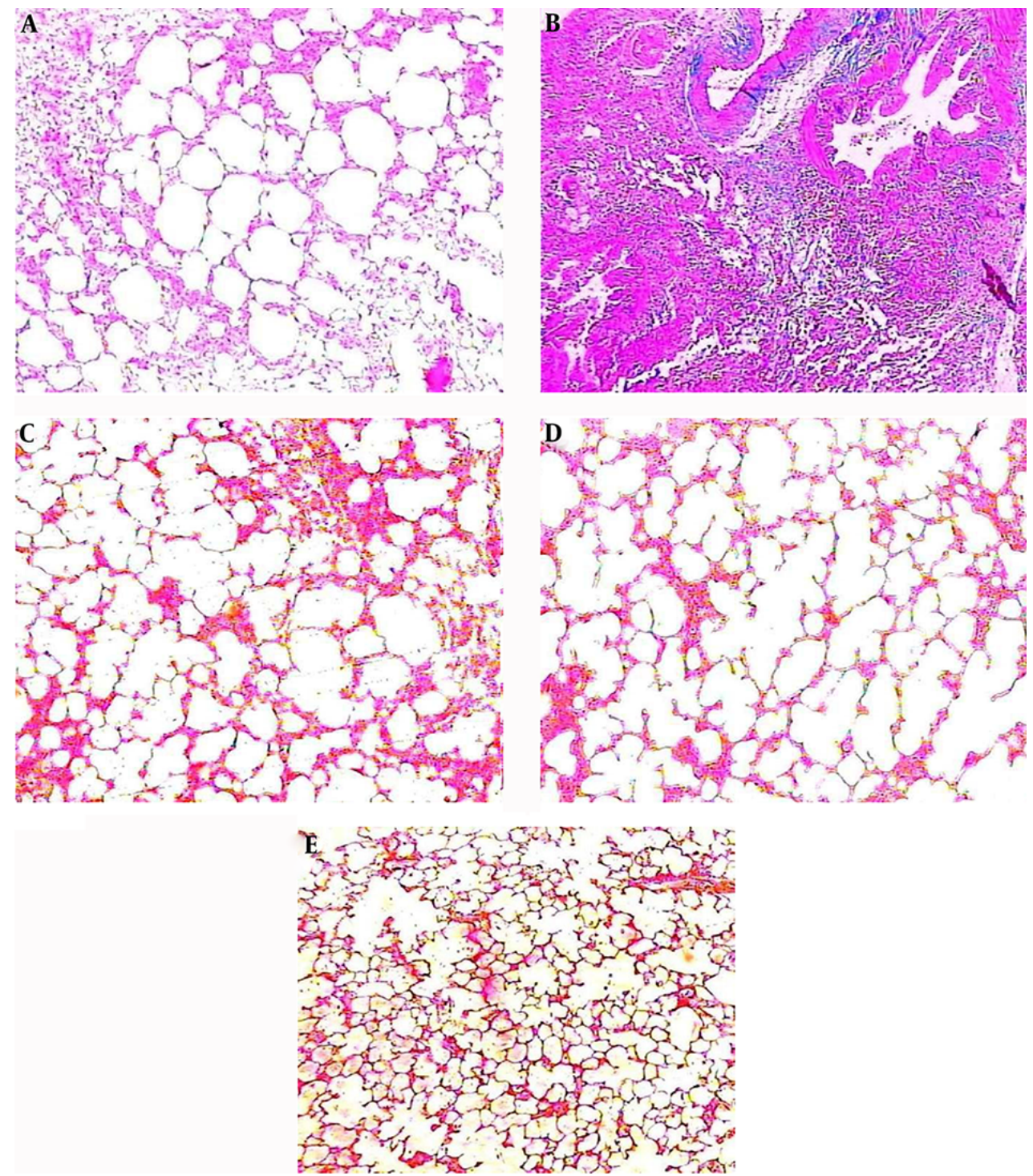

Figure 3. The Influence of valsartan on histopathological changes in rats with BLM-induced pulmonary fibrosis: A, control group; B, BLM group; C, valsartan (20 mg/kg) group; D, valsartan (40 mg/kg) group; E, valsartan ( $80 \mathrm{mg} / \mathrm{kg}$ ) group.

as a model for fibrosis of lungs in rats $(4,5)$.

In this analysis, we evaluated the anti-fibrotic effects of valsartan as an angiotensin receptor antagonist in bleomycin-induced pulmonary fibrosis. The present study showed that valsartan significantly decreased lung inflammatory infiltration after bleomycin exposure. Several studies have reported an anti-inflammatory effect of AT1 receptor blockade (17-19). An enhancement in lung hydroxyproline content (as a remarkable indicator of collagen quantity) was observed on the 21st day of treatment after bleomycin exposure in the bleomycin group. In the treatment groups, the hydroxyprolin content was markedly de- creased. Histopathological evaluation showed that valsartan noticeably reduced the extent and severity of the histological signs of tissue damage.

Corresponding in vitro studies have shown Ang II to be pro-fibrotic, thus causing an up-regulation of collagen gene expression in human lung fibroblasts (7). Ang II as a key mediator in the pathogenesis of lung fibrosis could influence the progression of lung injury via a number of mechanisms (17). Several previous investigations have reported an increase in lung Ang II concentrations and lung collagen content. Up-regulation in AT1 receptor expression occurred in lung parenchyma after bleomycin- 
induced lung injury. These studies' results suggest that angiotensinogen and AT1 receptors are expressed in lung tissue $(7,8,17)$. Previous assessments have also shown that AT1 receptor antagonists decrease lung collagen deposition, which was confirmed in our observations $(7,17,20)$. Valsartan could attenuate pulmonary fibrosis in both the treatment and pre-treatment stages. Our histological evaluation of the lung tissue and the reduction of hydroxyproline content indicated a protective effect of valsartan.

Our findings are in agreement with those of MolinaMolina et al. (21), who showed that losartan attenuated pulmonary fibrosis by increasing prostaglandin E2. Otsuka et al.'s (17) studies on candesartan indicated that candesartan could decrease the concentration of neutrophils in bronchoalveolar lavage (BAL) fluid in the bleomycin exposure group, which suggests that Ang II may play an important role in neutrophilic infiltration in the lung. Long et al. (22) showed an anti-fibrotic effect of valsartan via increased collagen synthesis through the induction of connective tissue growth factor (CTGF) expression. Yao et al. investigated the anti-fibrotic effect of losartan and found that the protective effect of losartan may be associated with its antifree radicals and the reduction in TGF- $\beta 1$ (23). The results of Ghazi-Khansari et al.'s work (24) showed that captopril and enalapril improved pulmonary fibrosis in histopathological studies as well as decreasing collagen content in the lung tissue. Marut et al.'s research (25) on irbesartan as an AT1 blocker indicated a protective effect of irbesartan by the inhibition of inflammation and NO production.

The data gathered in the current assessment lend support to the protective effect of valsartan as an angiotensin receptor blocker and provide evidence that further work should be conducted on angiotensin receptor antagonists as a promising treatment option for pulmonary fibrosis. Molecular and genetic studies are necessary to clarify the exact involvement mechanisms of the anti-fibrotic influences of valsartan.

\section{Acknowledgments}

This work was conducted as part of a PhD project in pharmacology financed by Ahvaz Jundishapur University of Medical Sciences. The authors wish to thank the university for its financial and instrumental support.

\section{Footnotes}

Authors' Contribution: Ali Asghar Hemmati designed the study protocol; Hoda Mojiri Forushani developed and performed the protocol and undertook the data analysis.
Funding/Support: This work was supported by the department of pharmacology and toxicology, Ahvaz University of Medical Sciences, Ahvaz, Iran.

\section{References}

1. Gribbin J, Hubbard RB, Le Jeune I, Smith CJ, West J, Tata LJ. Incidence and mortality of idiopathic pulmonary fibrosis and sarcoidosis in the UK. Thorax. 2006;61(11):980-5. doi:10.1136/thx.2006.062836. [PubMed: 16844727].

2. Selman M, Pardo A. Idiopathic pulmonary fibrosis: an epithelial/fibroblastic cross-talk disorder. Respir Res. 2002;3:3. [PubMed: 11806838].

3. Green FH. Overview of pulmonary fibrosis. Chest. 2002;122(6 Suppl):334-9. [PubMed: 12475811].

4. Ozyurt H, Sogut S, Yildirim Z, Kart L, Iraz M, Armutcu F, et al. Inhibitory effect of caffeic acid phenethyl ester on bleomycine-induced lung fibrosis in rats. Clin Chim Acta. 2004;339(1-2):65-75. [PubMed: 14687895].

5. Pardo A, Ruiz V, Arreola JL, Ramirez R, Cisneros-Lira J, Gaxiola $\mathrm{M}$, et al. Bleomycin-induced pulmonary fibrosis is attenuated in gamma-glutamyl transpeptidase-deficient mice. Am J Respir Crit Care Med. 2003;167(6):925-32. doi:10.1164/rccm.200209-1007OC. [PubMed: 12468440].

6. Shenoy V, Ferreira AJ, Qi Y, Fraga-Silva RA, Diez-Freire C, Dooies A, et al. The angiotensin-converting enzyme 2/angiogenesis-(1-7)/Mas axis confers cardiopulmonary protection against lung fibrosis and pulmonary hypertension. Am J Respir Crit Care Med. 2010;182(8):1065-72. doi: 10.1164/rccm.200912-18400C. [PubMed: 20581171].

7. Marshall RP, Gohlke P, Chambers RC, Howell DC, Bottoms SE, Unger $\mathrm{T}$, et al. Angiotensin II and the fibroproliferative response to acute lung injury. Am JPhysiol Lung Cell Mol Physiol. 2004;286(1):L156-64. doi: 10.1152/ajplung.00313.2002. [PubMed: 12754187].

8. Marshall RP, McAnulty RJ, Laurent GJ. Angiotensin II is mitogenic for human lung fibroblasts via activation of the type 1 receptor. Am J Respir Crit Care Med. 2000;161(6):1999-2004. doi: 10.1164/ajrccm.161.6.9907004. [PubMed: 10852780].

9. Papp M, Li X, Zhuang J, Wang R, Uhal BD. Angiotensin receptor subtype AT(1) mediates alveolar epithelial cell apoptosis in response to ANG II. Am J Physiol Lung Cell Mol Physiol. 2002;282(4):713-8. doi: 10.1152/ajplung.00103.2001. [PubMed: 11880296].

10. Li X, Rayford H, Uhal BD. Essential roles for angiotensin receptor AT1a in bleomycin-induced apoptosis and lung fibrosis in mice. Am J Pathol. 2003;163(6):2523-30. doi: 10.1016/S0002-9440(10)63607-3. [PubMed: 14633624 ].

11. Molteni A. Editorial Part-II: Applications of Angiotensin Converting Enzyme Inhibitors and of Angiotensin II Receptor Blockers in Pharmacology and Therapy: An Update. Curr Pharm Des. 2007;13(13):12958. [PubMed: 17506715].

12. Couluris M, Kinder BW, Xu P, Gross-King M, Krischer J, Panos RJ. Treatment of idiopathic pulmonary fibrosis with losartan: a pilot project. Lung. 2012;190(5):523-7. doi: 10.1007/s00408-012-9410-z. [PubMed: 22810758].

13. Black HR, Bailey J, Zappe D, Samuel R. Valsartan: more than a decade of experience. Drugs. 2009;69(17):2393-414. doi: 10.2165/11319460000000000-00000. [PubMed: 19911855].

14. Schraufnagel DE, Mehta D, Harshbarger R, Treviranus K, Wang NS. Capillary remodeling in bleomycin-induced pulmonary fibrosis. Am J Pathol. 1986;125(1):97-106. [PubMed: 2430459].

15. Woessner JFJ. The determination of hydroxyproline in tissue and protein samples containing small proportions of this imino acid. Arch Biochem Biophys. 1961;93:440-7. [PubMed: 13786180].

16. Lasky JA, Ortiz LA. Antifibrotic therapy for the treatment of pulmonary fibrosis. Am J Med Sci. 2001;322(4):213-21. [PubMed: 11678519]. 
17. Otsuka M, Takahashi H, Shiratori M, Chiba H, Abe S. Reduction of bleomycin induced lung fibrosis by candesartan cilexetil, an angiotensin II type 1 receptor antagonist. Thorax. 2004;59(1):31-8. [PubMed: 14694243].

18. Grafe M, Auch-Schwelk W, Zakrzewicz A, Regitz-Zagrosek V, Bartsch $\mathrm{P}$, Graf K, et al. Angiotensin II-induced leukocyte adhesion on human coronary endothelial cells is mediated by E-selectin. Circ Res. 1997;81(5):804-11. [PubMed: 9351454].

19. Ito $\mathrm{H}$, Takemori K, Suzuki T. Role of angiotensin II type 1 receptor in the leucocytes and endothelial cells of brain microvessels in the pathogenesis of hypertensive cerebral injury. J Hypertens. 2001;19(3 Pt 2):591-7. [PubMed: 11327634].

20. Uhal BD, Wang R, Laukka J, Zhuang J, Soledad-Conrad V, Filippatos G Inhibition of amiodarone-induced lung fibrosis but not alveolitis by angiotensin system antagonists. Pharmacol Toxicol. 2003;92(2):81-7. [PubMed: 12747577].

21. Molina-Molina M, Serrano-Mollar A, Bulbena O, Fernandez-Zabalegui L, Closa D, Marin-Arguedas A, et al. Losartan attenuates bleomycin in- duced lung fibrosis by increasing prostaglandin E2 synthesis. Thorax. 2006;61(7):604-10. doi: 10.1136/thx.2005.051946. [PubMed: 16601095].

22. Long X, Xiong SD, Xiong WN, Xu YJ, Zhang ZX, Cao Y, et al. Losartan inhibited angiotensin induced human lung fibroblast transformation and collagen synthesis [in Chinese]. Zhonghua Jie He He Hu Xi Za Zhi. 2007;30(4):273-8. [PubMed: 17651610].

23. Yao HW, Zhu JP, Zhao MH, Lu Y. Losartan attenuates bleomycininduced pulmonary fibrosis in rats. Respiration. 2006;73(2):236-42. doi: 10.1159/000090140. [PubMed: 16330873].

24. Ghazi-Khansari M, Mohammadi-Karakani A, Sotoudeh M, Mokhtary P, Pour-Esmaeil E, Maghsoud S. Antifibrotic effect of captopril and enalapril on paraquat-induced lung fibrosis in rats. J Appl Toxicol. 2007;27(4):342-9. doi: 10.1002/jat.1212. [PubMed: 17265423].

25. Marut W, Kavian N, Servettaz A, Hua-Huy T, Nicco C, Chereau C, et al Amelioration of systemic fibrosis in mice by angiotensin II receptor blockade. Arthritis Rheum. 2013;65(5):1367-77. doi: 10.1002/art.37873. [PubMed: 23335130]. 